\title{
Entropy of Bosonic Open String and Boundary Conditions
}

\author{
M. C. B. Abdalla ${ }^{1 *}$ E. L. Graça ${ }^{23 \dagger}$ I. V. Vancea ${ }^{12 \ddagger}$ \\ ${ }^{1}$ Instituto de Física Teórica, Universidade Estadual Paulista \\ Rua Pamplona 145, 01405-900 São Paulo-SP, Brasil \\ 2 Centro Brasileiro de Pesquisas Físicas \\ Rua Dr. Xavier Sigaud 150, CEP 22290-180 Rio de Janeiro RJ, Brasil \\ ${ }^{3}$ Departamento de Física \\ Universidade Federal Rural do Rio de Janeiro \\ BR 465 - 07 - Seropédica CEP 23890-000 RJ, Brasil
}

October 31, 2018

\begin{abstract}
The entropy of the states associated to the solutions of the equations of motion of the bosonic open string with combinations of Neumann and Dirichlet boundary conditions is given. Also, the entropy of the string in the states $\left|A^{i}\right\rangle=\alpha_{-1}^{i}|0\rangle$ and $\left|\phi^{a}\right\rangle=\alpha_{-1}^{a}|0\rangle$ that describe the massless fields on the world-volume of the $D p$-brane is computed.
\end{abstract}

\section{Introduction}

Recently, there has been some interest in formulating the $D$-branes at finite temperature for several reasons. On one hand, the relation between the $D$-branes and the field theory at finite temperature represents an interesting problem by itself which could help us to get a better understanding of the physical properties of $D$-branes. Some progress in this direction has been done in the low energy limit of string theory where the $D$-branes are solitonic solutions of (super)gravity. In this limit, the thermodynamics of $D$-branes has been formulated in the framework of (real

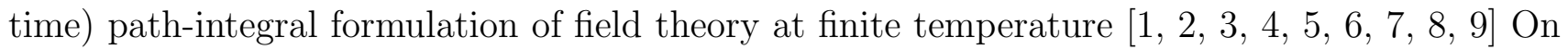
the other hand, one would like to understand the statistical properties of some systems that can

${ }^{*}$ Email: mabdalla@ift. unesp.br

${ }^{\dagger}$ Email: edison@cbpf.br 
be described in terms of $D$-branes and anti- $D$-branes as, for example, the extreme, near-extreme and Schwarzschild black-holes 110, 11, 12, 13, 14, 15, 16, 17, 18, 19, 20, 21, 22, 23, 24, 25, 26, 27.

In the other well understdood limit of string theory, the perturbative limit, the geometric information about the $D$-branes is lost. In that case, the $D$-branes are more appropriately described by a superposition of coherent states in the Fock space of closed string sector 28, 29, 30, 31, 32, 33, 34 that should satisfy a certain set of boundary conditions. They correspond to a combination of Dirichlet and Neumann boundary conditions which should be imposed at the endpoints of the open string in the open string channel. The intuitive interpretation of $D$-branes as coherent boundary states is maintained at finite temperature if one formulates the problem in the framework of the Thermo Field Dynamics (TFD). The reason for that is that the thermal dependence is implemented through the so called thermal Bogoliubov operators [35] which preserve the form of the relations at zero temperature. Working with TFD instead of real-time path integral formalism represent just a convenient way of formulating the problem since it is known that the two formalisms are equivalent at the thermodynamical equilibrium. The main idea of TFD is to interpret the statistical average of any quantity $Q$ over a statistical ensemble as the expectation value of $Q$ in a thermal vacuum

$$
Z^{-1}\left(\beta_{T}\right) \operatorname{Tr}\left[Q e^{-\beta_{T} H}\right]=\left\langle\left\langle 0\left(\beta_{T}\right)|Q| 0\left(\beta_{T}\right)\right\rangle\right\rangle
$$

where $\beta_{T}=\left(k_{B} T\right)^{-1}$ and $k_{B}$ is the Boltzmann's constant 35. The thermal vacuum is a vector in the direct product space of the original Fock space by an identical copy of it. The second copy of the Fock space provide the necessary states to describe the thermal effects.

The TFD was used to discuss the ideal gas of strings, to construct a bosonic open string field theory at finite temperature [36, 37, 38] and to prove its renormalizability [39, 40, 41]. These studies were motivated by the need of understanding string cosmology and the ensembles of strings in general. However, when one applies the TFD to D-branes one has to take some care 42, 43, 44, 45. Indeed, from the beginning, the $D$-branes are defined as states in the Fock space of the first quantized single string. The conformal field theory that underlies the construction describes the bosonic vacuum of string theory. When passing to finite temperature, one interprets the thermal string as a model for the thermal excitations of the bosonic vacuum of string theory. Therefore, the TFD is applied to the conformal field theory in two dimensions. Thus, the thermal $D$-branes should be interpreted as a coherent boundary states in the Fock space of the thermal excitations. Another remark is that according to (1), the entropy of the system is defined as the thermal vacuum expectation value of the entropy operator. However, our basic system is the

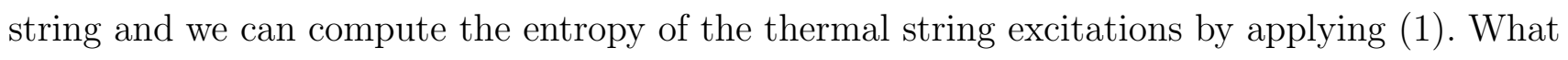
about the entropy of the $D$-brane states? At the moment, there is no known theory in which the $D$-branes are described by vacuum-like states or by states created from a vacuum. Thus, in order to compute the entropy of $D$-brane states, we have to calculate the average value of the 
our TFD approach to the thermal strings different from the previous ones.

The point of view adopted in [42, 43, 44, 45] is that of $D$-branes as states in the closed string sector. The TFD is applied to it. However, for the sake of completness, one should construct the thermal theory for the open string sector as well. In this case, one can wonder if the boundary conditions have any influence on the thermal properties of the system. The entropy of the open string in its termal vacuum is given by the sum over all space-time directions of the entropy of the scalar fields and thus it does not depend on the boundary conditions. However, if the string is in a state that depends on the boundary conditions, the entropy computed as the average of the entropy operator in that state depends on them. Since the bosonic vacuum depends on what sort of boundary conditions we impose to the equations of motion (for example, the Dirichelt boundary conditions will indicate the presence of branes), we would like to look to the entropy in the states associated to the general solutions of the equations of motion with a mixture of Dirichlet and Neumann boundary conditions. The aim of the present paper is to present the TFD construction for the bosonic string in the sense explained above, i. e. for bosonic string vacuum, and to compute its entropy in states with explicit dependence on boundary conditions. In particular, we calculate the entropy of open strings in the thermal massless states which can be interpreted as thermal massless fields living on the $D$-brane. There are at least two reasons for which such of computation should be performed. Firstly, it represents a natural step in developing a theory of string vacua and thermal branes in TFD approach. In this context, the states that present an explicit dependence on the boundary states play a fundamental role. Secondly, it might help to understand the entropy of the $D$-branes in the perturbative limit of string theory.

\section{The Bosonic String in TFD}

Consider a bosonic open string in flat spacetime. In order to avoid the introduction of ghosts, we choose to work in the light-cone gauge $X^{0} \pm X^{9}$ so that the spacetime indices run over the set $\mu, \nu=1,2, \cdots, 24$. The string can be quantized in the canonical formalism by interpreting the most general solution of the equations of motion as an operator on the Fock space and by imposing the canonical commutation relations. The dependence on the world-sheet parameters $(\tau, \sigma)$ is determined by the boundary conditions which can be of the Neumann $(\mathrm{N})$ or of the Dirichlet type, independently at each end of the string. Let us consider the string with NN boundary conditions. The other cases are treated in exactly the same manner. The general solutions $X^{\mu}(\tau, \sigma)$ of the equations of motion of the bosonic open string with the Neumann boundary conditions at the two ends are giving by the following relations

$$
X^{\mu}(\tau, \sigma)=x^{\mu}+2 \alpha^{\prime} \tau p^{\mu}+i \sqrt{2 \alpha^{\prime}} \sum_{n \neq 0} \frac{\alpha_{n}^{\mu}}{n} e^{-i n \tau} \cos n \sigma
$$


Here, $x^{\mu}$ and $p^{\mu}$ are the the canonical coordinates and momenta of the center of mass of the string. It is useful to introduce the oscillator operators

$$
A_{n}^{\mu}=\frac{1}{\sqrt{n}} \alpha_{n}^{\mu} \quad ; \quad A_{n}^{\mu \dagger}=\frac{1}{\sqrt{n}} \alpha_{-n}^{\mu} \quad, \quad n>0,
$$

that satisfy the canonical commutation relations among themselves and commute with the coordinates and momenta of the center of mass. The vacuum is defined to be invariant under translations

$$
|\Omega\rangle=|0\rangle|p\rangle
$$

where

$$
\begin{aligned}
A_{n}^{\mu}|0\rangle & =0, \quad \forall n \\
\hat{p}^{\mu}|p\rangle & =p^{\mu}|p\rangle .
\end{aligned}
$$

Now let us construct the thermal bosonic string. According to TFD, firstly one has to duplicate the system. The identical copy of it, denoted by, must be independent of the original string 42, 43, 44, 35]. The Hilbert space of the total system is the tensor product of the two Hilbert spaces $\mathcal{H}$ and $\tilde{\mathcal{H}}$. The operators for the two strings commute among themselves. By duplicating the system, new degrees of freedom are associated to the string. They provide enough room to accomodate the thermal properties of the bosonic vacuum of string. To this end, the new copy of the string cannot represent a physical system. Otherwise, the tilde Hilbert space will describe just the dynamical degrees of freedom of the second string, and no degrees of freedom will be left for the thermal effects. I They can be implemented in the enlarged Hilbert space by constructing a map called the thermal Bogoliubov transformation that maps the vacuum of the string to the thermal vacuum defined by (1). It turns out that there are several possibilities to construct such of map and that the corresponding operators generate a thermal $S U(1,1)$ group. But if we stick to unitary and tilde invariant transformations we are left with just a single operator in the present case [45, 46]. The Bogoliubov transformation is defined for each oscillator $n$ in each direction $\mu$ by the following relation

$$
G_{n}^{\mu}=-i \theta_{n}\left(\beta_{T}\right)\left(A_{n} \cdot \tilde{A}_{n}-\tilde{A}_{n}^{\dagger} \cdot A_{n}^{\dagger}\right)
$$

Here, $\theta_{n}\left(\beta_{T}\right)$ is a parameter related to the Bose-Einstein distribution of the oscillator $n$. The dot in (可) represents the Euclidean scalar product in the target space. The vacuum of the oscillators at $T \neq 0$ is given by the following relation

$$
\left.\left.\left|0\left(\beta_{T}\right)\right\rangle\right\rangle=\prod_{m>0} e^{-i G_{m}}|0\rangle\right\rangle,
$$

\footnotetext{
${ }^{1}$ We would like to thank to A. L. Gadelha for illuminating discussions on this point.
} 
where $|0\rangle\rangle=|0\rangle|\tilde{0}\rangle$ and

$$
G_{n}=\sum_{\mu=1}^{24} G_{n}^{\mu} .
$$

The total vacuum at $T \neq 0$ is constructed in the same way as (丑), i.e.

$$
\left.\left.\left|\Omega\left(\beta_{T}\right)\right\rangle\right\rangle=\left|0\left(\beta_{T}\right)\right\rangle\right\rangle|p\rangle|\tilde{p}\rangle .
$$

The state (8) is called the thermal vacuum since it is annihilated by all thermal annihilation operators constructed by acting with the Bogoliubov operators (9) onto the oscillator operators of string

$$
A_{n}^{\mu}\left(\beta_{T}\right)=e^{-i G_{n}} A_{n}^{\mu} e^{i G_{n}} \quad, \quad \tilde{A}_{n}^{\mu}\left(\beta_{T}\right)=e^{-i G_{n}} \tilde{A}_{n}^{\mu} e^{i G_{n}} .
$$

The same action of the Bogoliubov operators gives any other thermal operator. In particular, one constructs the creation operators $A_{n}^{\mu \dagger}\left(\beta_{T}\right)$ and $\tilde{A}_{n}^{\mu \dagger}\left(\beta_{T}\right)$ which, together with (11) define the set of thermal string oscillators since they satisfy the same commutation relations as the operators at $T=0$. The coordinates and the momenta of the center of mass of string are invariant under the Bogoliubov map. Thus, one can construct the string solution $X^{\mu}\left(\beta_{T}\right)$ at $T \neq 0$ by replacing the operators in (2) with the corresponding operators at $T \neq 0$. In exactly the same way one can construct the generators of the Virasoro algebra in terms of thermal oscillator operators and show that the conformal symmetry of the solution is preserved. Consequently, the Bogoliubov transformation maps the two string copies into two thermal string copies 43 , 44. However, the interpretation in terms of original and tilde string modes is lost at non-zero temperature since the Bogoliubov operators (17) mix the two copies of strings. Also, the thermal vacuum should be invariant under the tilde operation and therefore the tilde and non-tilde zero-temperature excitations are generated simultaneously at $T \neq 0$. This is an intrinsic fact of the TFD construction. Heuristically, one would say that the thermal string excitations, being a mixture of zero-temperature string and tilde string excitations, carry thermal degrees of freedom beside dynamical degrees of freedom (in zero-temperature language) which is in agreement with our intuition.

\section{The Entropy in States with Boundary Dependence}

Since we are treating the string as a set of bosonic oscillators, the entropy operators for the bosonic open string follow directly from the definition [35]

$$
\begin{aligned}
K & =\sum_{\mu=1}^{24} \sum_{n=1}^{\infty}\left(A_{n}^{\mu \dagger} A_{n}^{\mu} \log \sinh ^{2} \theta_{n}-A_{n}^{\mu} A_{n}^{\mu \dagger} \log \cosh ^{2} \theta_{n}\right) \\
\tilde{K} & =\sum_{\mu=1}^{24} \sum_{n=1}^{\infty}\left(\tilde{A}_{n}^{\mu \dagger} \tilde{A}_{n}^{\mu} \log \sinh ^{2} \theta_{n}-\tilde{A}_{n}^{\mu} \tilde{A}_{n}^{\mu \dagger} \log \cosh ^{2} \theta_{n}\right)
\end{aligned}
$$


The vacuum of the present theory is invariant under the tilde operation and all the physical information is contained in the operators without tilde. Therefore, we are going to calculate the matrix elements only of the entropy (12). The basic idea is to compute firstly the matrix elements to the contribution to the entropy of the excitations in each direction of space-time. Thus, it is useful to write (12) in the form

$$
K=\sum_{\mu=1}^{24} K^{\mu},
$$

where the notation is obvious.

The entropy of the string, as given by the formula (四), represents the sum of the entropy of all oscillators and it is straight-forward to write it down. Next, we would like to know the entropy of the string in states associated to the most general solution of the equation of motion. This is a similar situation to computing the entropy of $D$-branes. In this case, the entropy is a world-sheet function and its dependence on the world-sheet parameters is dictated by the boundary conditions imposed on the string equations of motion. Upon quantization, the general solution of the string equations of motion with given boundary conditions at the two ends becomes an operator acting on the thermal Fock space. The state $\left.\left|X^{\mu}\left(\beta_{T}\right)\right\rangle\right\rangle$ obtained by applying (2) on the thermal vacuum (10) can be associated to it. Let us work out the matrix elements $\left\langle\left\langle X^{\mu}\left(\beta_{T}\right)\left|K^{\rho}\right| X^{\mu}\left(\beta_{T}\right)\right\rangle\right\rangle$ for the NN boundary conditions in some detail. The computations envolving the other boundary conditions follow the same line.

The matrix element $\left\langle\left\langle X^{\mu}\left(\beta_{T}\right)\left|K^{\rho}\right| X^{\mu}\left(\beta_{T}\right)\right\rangle\right\rangle$ can be split in two parts: one part containing only the oscillator contribution and a part that contains the coordinates and the momenta of the center of mass

$$
\begin{aligned}
\left\langle\left\langle X^{\mu}\left(\beta_{T}\right)\left|K^{\rho}\right| X^{\mu}\left(\beta_{T}\right)\right\rangle\right\rangle & =\mathrm{CM} \text { terms } \\
& -2 \alpha^{\prime} \sum_{n, k, l>0} \frac{e^{i(l-n) \tau}}{\sqrt{l n}} \cos n \sigma \cos l \sigma\left[\left(T_{1}\right)_{n k l}^{\mu \rho \nu}+\left(T_{2}\right)_{n k l}^{\mu \rho \nu}\right]
\end{aligned}
$$

where

$$
\begin{aligned}
\left(T_{1}\right)_{n k l}^{\mu \rho \nu} & =\left\langle\tilde{0}\left|\left\langle 1_{n}^{\mu}\left|\prod_{m>0} e^{-i G_{m}} A_{k}^{\rho \dagger} A_{k}^{\rho} \log \sinh ^{2} \theta_{k} \prod_{s>0} e^{i G_{s}}\right| 1_{l}^{\nu}\right\rangle\right| \tilde{0}\right\rangle\langle\tilde{p} \mid \tilde{q}\rangle\langle p \mid q\rangle, \\
\left(T_{2}\right)_{n k l}^{\mu \rho \nu} & =-\left\langle\tilde{0} \mid\left\langle 1_{n}^{\mu}\left|\prod_{m>0} e^{-i G_{m}} A_{k}^{\rho} A_{k}^{\rho \dagger} \log \cosh ^{2} \theta_{k} \prod_{s>0} e^{i G_{s}}\right| 1_{l}^{\nu}\right\rangle \tilde{0}\right\rangle\langle\tilde{p} \mid \tilde{q}\rangle\langle p \mid q\rangle
\end{aligned}
$$

and

$$
\left|1_{l}^{\mu}\right\rangle=A_{l}^{\mu \dagger}|0\rangle
$$

We consider the usual normalization of the momentum states in a finite volume $V_{24}$ in the transverse space

$$
\langle p \mid q\rangle=2 \pi \delta^{(24)}(p-q)
$$


It follows from a simple algebra that the pure oscillator contribution to the matrix element of the entropy operator has the following form

$$
\begin{aligned}
\left\langle\left\langle X^{\mu}\left(\beta_{T}\right)\left|K^{\rho}\right| X^{\mu}\left(\beta_{T}\right)\right\rangle\right\rangle= & \mathrm{CM} \text { terms }-2 \alpha^{\prime}(2 \pi)^{(48)} \delta^{\mu \nu} \delta^{(24)}(p-q) \delta^{(24)}(\tilde{p}-\tilde{q}) \times \\
& \sum_{n>0} \frac{1}{n} \cos ^{2} n \sigma\left[\log \left(\tanh \theta_{n}\right)^{2} \delta^{\rho \nu}-\delta^{\rho \rho} \sum_{k>0} \delta_{k k}\right] .
\end{aligned}
$$

The terms containing the center of mass can be divided at their turn in to two sets: the ones containing only the coordinates and momenta operators and the ones containing the contributions from the oscillators. The terms in coordinates and momenta can be calculated by using the completness relation of the eigenstates of momenta operators $|x\rangle$ together with the coordinate-momenta matrix

$$
\langle x \mid p\rangle=(2 \pi \hbar)^{-12} e^{i p \cdot x / \hbar} .
$$

In order to compute the terms containing oscillator contribution one can either express the oscillator operators at zero temperature in terms of operators at $T \neq 0$ or write the vacuum at $T \neq 0$ in terms of vacuum at zero temperature. The two ways lead to the same result, however in the first case one has to deal only with polynomial relations in creation and annihilation operators at finite temperature. Using the properties of the Bogoliubov operators deduced in [43, 44] and after a simple algebra one can show that the contribution coming from terms that mix the center of mass operators with the oscillator part cancells. The non-vanishing terms are all proportional to $\left\langle\left\langle 0\left(\beta_{T}\right)\left|K^{\rho}\right| 0\left(\beta_{T}\right)\right\rangle\right\rangle$ which represents the entropy of an infinite number of bosonic oscillators in the $\rho$ 'th directions of space-time. Putting all the results together we obtain the following relation

$$
\begin{aligned}
& \left\langle\left\langle X^{\mu}\left(\beta_{T}\right)\left|K^{\rho}\right| X^{\mu}\left(\beta_{T}\right)\right\rangle\right\rangle= \\
- & (2 \pi \hbar)^{-24}\left[(2 \pi \hbar)^{24}\left(2 \alpha^{\prime} \tau\right)^{2} p^{\mu} p^{\nu} \delta^{(24)}\left(p-p^{\prime}\right)+2 \alpha^{\prime} \tau\left(I_{2}^{\mu} p^{\prime \nu}+I_{2}^{\prime \nu} p^{\mu}\right)+I_{2}^{\mu} I_{2}^{\nu} \prod_{j \neq \mu, \nu} I_{1}^{j}\right] \\
\times & \delta^{(24)}\left(\tilde{p}-\tilde{p^{\prime}}\right) \sum_{m=1}\left[\mathrm{n}_{m}^{\rho} \log \mathrm{n}_{m}^{\rho}+\left(1-\mathrm{n}_{m}^{\rho}\right) \log \left(1-\mathrm{n}_{m}^{\rho}\right)\right]-2 \alpha^{\prime}(2 \pi)^{(48)} \delta^{\mu \nu} \delta^{(24)}\left(p-p^{\prime}\right) \\
\times & \delta^{(24)}\left(\tilde{p}-\tilde{p^{\prime}}\right) \sum_{n>0} \frac{1}{n} \cos ^{2} n \sigma\left[\log \left(\tanh \theta_{n}\right)^{2} \delta^{\rho \nu}-\delta^{\rho \rho} \sum_{k>0} \delta_{k k}\right],
\end{aligned}
$$

where the unidimensional integrals on the finite domains $x \in\left[x_{0}, x_{1}\right]$ are given by

$$
\begin{aligned}
& I_{1}=-i \hbar\left(p^{\prime}-p\right)^{-1}\left[e^{\frac{i}{\hbar}\left(p^{\prime}-p\right) x_{1}}-e^{\frac{i}{\hbar}\left(p^{\prime}-p\right) x_{0}}\right] \\
& I_{2}=-i \hbar\left(p^{\prime}-p\right)^{-1}\left[-i \hbar I_{1}+x_{1} e^{\frac{i}{\hbar}\left(p^{\prime}-p\right) x_{1}}-x_{0} e^{\frac{i}{\hbar}\left(p^{\prime}-p\right) x_{0}}\right] .
\end{aligned}
$$

Here, the momentum of the final and initial states are denoted by $|p\rangle$ and $\left|p^{\prime}\right\rangle$, respectively and

$$
\mathrm{n}_{\mathrm{m}}^{\rho}=\left\langle\left\langle 0\left(\beta_{T}\right)\left|A_{m}^{\rho \dagger} A_{m}^{\rho}\right| 0\left(\beta_{T}\right)\right\rangle\right\rangle=\sinh ^{2} \theta_{m}
$$


represents the number of string excitations in the thermal vacuum.

Relation (22) represents the matrix element of the entropy operator $K^{\rho}$ between two general states described by solutions of the equations of motion of the open bosonic string with NN boundary conditions. The total energy is a sum over all directions in the transverse space. Since the effect of the boundary condition appears only in the dependence on the world-sheet parameters, it is easy to write down the similar relations for the other boundary conditions DD, DN and ND. In these cases, there are no operators associated with the coordinate and momenta of the center of mass of the open string but rather constant position vectors associated to the endpoints of string. Therefore, there is no contributions from these terms to the entropy. The terms that mix the coordinates of the endpoints with the oscillators vanish for the same reason as in the NN case. The only non-vanishing terms in the matrix elements of the entropy are

$$
\begin{array}{rlrl}
\mathrm{D} D & : & \left\langle\left\langle X^{\mu}\left(\beta_{T}\right)\left|K^{\rho}\right| X^{\mu}\left(\beta_{T}\right)\right\rangle\right\rangle=2 \alpha^{\prime}(2 \pi)^{(48)} \delta^{\mu \nu} \delta^{(24)}\left(p-p^{\prime}\right) \delta^{(24)}\left(\tilde{p}-\tilde{p^{\prime}}\right) \\
& \times \sum_{n>0} \frac{1}{n} \sin ^{2} n \sigma\left[\log \left(\tanh \theta_{n}\right)^{2} \delta^{\rho \nu}-\delta^{\rho \rho} \sum_{k>0} \delta_{k k}\right] \\
\mathrm{D} N & : \quad\left\langle\left\langle X^{\mu}\left(\beta_{T}\right)\left|K^{\rho}\right| X^{\mu}\left(\beta_{T}\right)\right\rangle\right\rangle=2 \alpha^{\prime}(2 \pi)^{(48)} \delta^{\mu \nu} \delta^{(24)}\left(p-p^{\prime}\right) \delta^{(24)}\left(\tilde{p}-\tilde{p^{\prime}}\right) \\
& \times \sum_{r=\mathcal{Z}+1 / 2} \frac{1}{r} \sin ^{2} r \sigma\left[\log \left(\tanh \theta_{r}\right)^{2} \delta^{\rho \nu}-\delta^{\rho \rho} \sum_{k>0} \delta_{k k}\right] \\
\mathrm{N} D & : \quad\left\langle\left\langle X^{\mu}\left(\beta_{T}\right)\left|K^{\rho}\right| X^{\mu}\left(\beta_{T}\right)\right\rangle\right\rangle=2 \alpha^{\prime}(2 \pi)^{(48)} \delta^{\mu \nu} \delta^{(24)}\left(p-p^{\prime}\right) \delta^{(24)}\left(\tilde{p}-\tilde{p^{\prime}}\right) \\
& \times \sum_{r=\mathcal{Z}+1 / 2} \frac{1}{r} \cos ^{2} r \sigma\left[\log \left(\tanh \theta_{r}\right)^{2} \delta^{\rho \nu}-\delta^{\rho \rho} \sum_{k>0} \delta_{k k}\right]
\end{array}
$$

Here, $\mathcal{Z}+1 / 2$ are the half-integer numbers. The contribution of just a single field is obtained by taking $\mu=\rho=\nu$. The relations (22), (26), (27) and (28) represent the entropy of the states associated to the general solutions of the equations of motion. They give the entropy as a function on the world-sheet, therefore it should not be taken for the entropy of the bosonic string vacuum which is simply the sum over all space-time directions of the entropy of massless scalar bosons and it does not depend on the boundary conditions as it should.

\section{Discussions}

The matrix elements (22), (26), (27) and (28) can be used to calculate the entropy of various states of open strings with different boundary conditions. As an example, consider the massless states of strings ending on one $D p$-brane. It is known that these states describe massless fields on the world-volume of the brane. They form an $U(1)$ multiplet $A^{j}=\alpha_{-1}^{j}|0\rangle$, where $j=1,2, \ldots, p$ and a set of $(24-p)$ scalars $\phi^{a}=\alpha_{-1}^{a}|0\rangle$ where $a=p+1, \ldots, 24$. The contribution to the entropy of the corresponding string states with DD, DN and ND boundary condition can be 
elements of $K^{\rho}$ in the thermal states

$$
\left.\left.\left|\Psi^{\lambda}\left(\beta_{T}\right)\right\rangle\right\rangle=\alpha_{-1}^{\lambda}\left(\beta_{T}\right)\left|0\left(\beta_{T}\right)\right\rangle\right\rangle
$$

multiplied by the function on $\tau$ and $\sigma$ that is obtained from the corresponding boundary conditions. A simple algebra gives the follwing expression for the entropies of the $U(1)$ multiplet and the set of scalars, respectively

$$
\begin{aligned}
& E_{\{A\}}=2 \alpha^{\prime} p\left(-\sin ^{2} \sigma \sum_{n=1} \log \cosh ^{2} \theta_{n}+4 \cos ^{2} \sigma \sum_{r \in \mathcal{Z}+1 / 2} \log \cosh ^{2} \theta_{r}\right), \\
& E_{\{\phi\}}=2 \alpha^{\prime}(24-p)\left(-\sin ^{2} \sigma \sum_{n=1} \log \cosh ^{2} \theta_{n}+4 \cos ^{2} \sigma \sum_{r \in \mathcal{Z}+1 / 2} \log \cosh ^{2} \theta_{r}\right) .
\end{aligned}
$$

In (30) and (31) the first term represents the contribution from the DD sector while the second one is obtained from DN and ND sectors.

In conclusion, we have calculated the entropy of string in states that depend explicitely on the boundary conditions. To this end, we have picked up the most general solution of the equations of motion with all possible combinations of Dirichlet and Neumann boundary conditions and we computed the matrix elements of the entropy in the state associated to it. The relevant relations are (22), (26), (27) and (28). It is interesting to observe that only the NN entropy depends on $\hbar$. In the semiclassical limit $\hbar \rightarrow 0$ the pure momentum contribution becomes irrelevant. The dominating term is the same that dominates the infinite tension limit $\alpha^{\prime} \rightarrow 0$. In this case the entropy of the DD, DN and ND strings vanishes.

In particular, we have applied the general results to the computation of the entropy of the massless string states that generate the field theories on the world-volume of a $D p$-brane. In the case of a stack of $N$ parallel D-branes the gauge multiplet is of $U(N)$ and in order to find the total entropy of it we just have to sum over the internal indices. However, the scalars become non-commutative and the present approach should be extended to include non-commutative fields [49]. Constructing the $D$-branes in the thermal string field theory represents a different but interesting topic. If such of theory were given, one could get insights in the problem of the entropy of the $D$-brane which, in the present formulation, seems to suffer from certain anomalies 44.

Acknowledgements We would like to thank N. Berkovits, A. L. Gadelha, P. K. Panda, B. M. Pimentel, H. Q. Placido and W. P. de Souza for useful discussions. I. V. V. also acknowledge to S. A. Dias and J. A. Helayël-Neto for hospitality at DCP-CBPF and GFT-UCP where part of this work was done. I. V. V. was supported by a FAPESP postdoc fellowship.

\section{References}


[1] M. V. Mozo, Phys. Lett. B388(1996)494; J. L. F. Barbon and M. V. Mozo, Nucl. Phys. B497(1997)236

[2] A. Bytsenko, S. Odintsov and L. Granada, Mod. Phys. Lett. A11(1996)2525

[3] J. Ambjorn, Yu. Makeenko, G. W. Semenoff and R. Szabo, Phys. Rev. D60(1999)106009

[4] G. Dvali, I. I. Kogan and M. Shifman, Phys. Rev. D62(2000)106001

[5] S. Abel, K. Freese and I. I. Kogan, JHEP 0101(2001)039

[6] I. I. Kogan, A. Kovner and M. Schvellinger, hep-th/0103235

[7] S. A. Abel, J. L. F. Barbon, I. I. Kogan, E. Rabinovici, JHEP 9904(1999)015

[8] J. L. F. Barbon, E. Rabinovici, JHEP 0106(2001)029

[9] S. S. Gubser, I. R. Klebanov, M. Rangamani, E. Witten, hep-th/0009140

[10] A. Sen, Nucl. Phys. B440(1995)421

[11] A. Strominger and C. Vafa, Phys. Lett. B379(1996)99

[12] L. Susskind, hep-th/9309145

[13] G. Horowitz and J. Polchinski, Phys. Rev. D55(1997)6189

[14] T. Banks, W. Fischler, I. Klebanov and L. Susskind, Phys. Rev. Lett. 80(1998)226, JHEP 9801(1998)008

[15] S. Chaudhuri and D. Minic, Phys. Lett. B433(1998)301

[16] I. Klebanov and L. Susskind, Phys. Lett. B416(1998)62

[17] E. Halyo, hep-th/9709225

[18] S. Das, S. Mathur, S. Kalyana Rama and P. Ramadevi, Nucl. Phys. B527(1998)187

[19] G. Horowitz and E. Martinec, Phys. Rev. D57(1998)4935

[20] M. Li, JHEP 9801(1998)009; M. Li and E. Martinec, hep-th/9801070

[21] T. Banks, W. Fischler and I. Klebanov, Phys. Lett. B423(1998)54

[22] H. Liu and A. Tseytlin, JHEP 9801(1998)010

[23] D. Minic, hep-th/9712202 
[24] A. Strominger, Phys. Rev. Lett. 71(1993)3397

[25] I. V. Volovich, hep-th/9608137

[26] J. Maldacena and A. Strominger, JHEP 9807(1998)013

[27] U. H. Danielsson, A. Guijosa, M. Kruckzenski, JHEP0109(2001)011

[28] M. B. Green and P. Wai, Nucl. Phys. B431(1994)131

[29] M. B. Green, Nucl. Phys. B381(1992)201

[30] M. B. Green and M. Gutperle, Nucl.Phys. B476(1996)484

[31] M. Li, Nucl. Phys. B 460(1996)351

[32] C. Callan Jr. and I. Klebanov, Nucl. Phys. B465(1996)473

[33] P. Di Vecchia and A. Liccardo, D-Branes in String Theory, hep-th/9912161, hep-th/9912275

[34] P. Di Vecchia, M. Frau, A. Lerda and A. Liccardo, Nucl. Phys. B565(2000)397

[35] Y. Takahashi and H. Umezava, Collective Phenom. 2(1975)55

[36] Y. Leblanc, Phys. Rev. D38(1988)3087

[37] Y. Leblanc, Phys. Rev. D36(1987)1780; Phys. Rev. D37(1988)1547; Phys. Rev. D39(1989)1139; Phys. Rev. D39 (1989)3731

[38] Y. Leblanc, M. Knecht and J. C. Wallet, Phys. Lett. B237 (1990)357

[39] H. Fujisaki and K. Nakagawa, Prog. Theor. Phys. 82(1989)236; Prog. Theor. Phys. 82(1989)1017; Prog. Theor. Phys. 83(1990)18; Europhys. Lett. 20(1992)677; Europhys. Lett. 28(1994) 471

[40] H. Fujisaki, Il Nuovo Cimento, 108A(1995)1079

[41] H. Fujisaki and K. Nakagawa, Europhys. Lett. 35(1996)493

[42] M. B. C. Abdalla, A. L. Gadelha and I. V. Vancea, Phys. Lett. A273(2000)235

[43] I. V. Vancea, Phys. Lett. B487(2000)175

[44] M. B. C. Abdalla, A. L. Gadelha and I. V. Vancea, Phys. Rev. D64(2001)086005

[45] M. B. C. Abdalla, A. L. Gadelha and I. V. Vancea, On The Thermal SU $(1,1)$ Group for Bosonic Strinas and D-branes in preparation 
[46] H. Umezawa, Advanced Field Theory: Micro, Macro and Thermal Field(American Institute of Physics, 1993)

[47] M. J. Bowick and L. C. R. Wiejewardhana, Phys. Rev. Lett. 25(1970)895

[48] B. Sunborg, Nucl. Phys. B254(1985)583

[49] M. C. B. Abdalla, A. L. Gadelha, E. L. Graça and I. V. Vancea, in preparation. 